\title{
Los Usos de Facebook y WhatsApp en la Reconstrucción de la Educación en Zonas Afectadas por Conflictos Armados: El Caso de Siria
}

\author{
Using Facebook and WhatsApp in Rebuilding the Education in \\ Areas Affected by Armed Conflicts: The Syrian Case
}

Mirey Alfarah *

Alejandra Bosco

Universidad Autónoma de Barcelona, España

\begin{abstract}
Desde el inicio del conflicto en Siria en 2011 más del 45\% de la población se ha visto forzada al desplazamiento, de la cual casi la mitad está compuesta por niños y niñas. Para una gran parte de la población siria, el acceso a la educación se ha visto interrumpido. Las estadísticas muestran que casi tres millones de niños y niñas sirios no han tenido acceso a ningún tipo de educación durante más de cuatro años. Los conflictos armados pueden perturbar o destruir totalmente las condiciones necesarias para asegurar un entorno sano, estable y seguro para la educación. El presente artículo muestra una parte de los resultados de una investigación en curso sobre las oportunidades que ofrecen las Tecnologías de la Información y de la Comunicación (TIC) en la reconstrucción de la educación en países afectados por conflictos armados, en particular, en el caso de Siria. Este trabajo se centra en el uso de las redes sociales (Facebook) y la mensajería instantánea (WhatsApp). La investigación desarrollada es un estudio de casos múltiples de carácter cualitativo. El análisis de los datos muestra que Facebook y WhatsApp mejoran el rendimiento de las organizaciones e instituciones cuyo objetivo es ofrecer una mejora a la crisis de la educación siria en tres aspectos principales: en el proceso de enseñanza y aprendizaje, en la comunicación, coordinación e información y en la recopilación y gestión de datos.
\end{abstract}

Descriptores: Tecnología de la comunicación; Educación; Redes sociales; Conflicto armado; Siria.

Since the beginning of the conflict in Syria in 2011 more than $45 \%$ of the population have been forced to displace, half of them being children. For a large part of the Syrian population, the access to education has been interrupted and statistics show that almost three million children were deprived of any type of education during a period of more than four years. Armed conflicts can disrupt or totally destroy the conditions necessary to ensure a healthy, stable and safe environment for education. This article reports part of the results of an investigation concerning the opportunities offered by Information and Communication Technologies (ICT) in rebuilding the education in countries affected by armed conflicts. The ongoing conflict in Syria is chosen as a particular case study. Concretely, this work focuses on the use of social networks (Facebook) and instant messaging (WhatsApp). The research is multi-case study based on the qualitative method. The analysis of the collected data indicates that Facebook and WhatsApp improve the performance of organizations and institutions whose objective is to offer a solution to the Syrian educational crisis regarding three main aspects: (1) in the teaching and learning process, (2) in communication, coordination and information and (3) in data collection and management.

Keywords: Communication technology; Education; Social networks; Armed conflicts; Syria.

\footnotetext{
*Contacto: mirey.alfarah@e-campus.uab.cat Recibido: 1 de junio 2018

$1^{\text {a }}$ Evaluación: 3 de julio 2018

ISSN: $1696-4713$

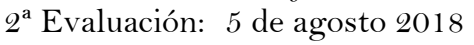

www.rinace.net/reice/

Aceptado: 27 de agosto 2018
}

revistas.uam.es/reice 


\section{Introducción}

Desde el inicio del conflicto en Siria en 2011, la infancia de este país padece las graves consecuencias que ha provocado el conflicto. El porcentaje de niños y niñas fuera de la escuela se ha triplicado de 0,9 millones (14\%) en el año académico 2011/12 a 2,8 millones (40\%) en el año académico 2014/15 (No Lost Generation, 2016; UNICEF, 2016b). Muchos niños y niñas sirios en edad escolar nunca han visto el interior de un aula y los que todavía tienen acceso a la educación siguen estando en riesgo de abandonar la escuela. Debido al conflicto, estos niños y niñas han sido privados de su infancia además de su derecho a una educación adecuada y de calidad. A fecha de hoy, hay 5,3 millones de niños y niñas sirios que no satisfacen sus necesidades básicas: asistencia sanitaria, protección infantil, educación, alimentación, seguridad y estabilidad (UNICEF, 2017).

Todo ello supone un impacto negativo en su estabilidad psicológica, desarrollo y oportunidades para el futuro, y plantea preocupaciones acerca de lo que podría denominarse una "generación perdida" (UNICEF, 2013). En consecuencia, surge la necesidad de crear soluciones a la crisis educativa siria. No obstante, no es una tarea fácil. La persistencia del conflicto, la incapacidad económica de los padres y madres, el trabajo infantil, la falta de espacio y recursos en las escuelas, las barreras lingüísticas y la discriminación en las escuelas de los países de acogida, son ejemplos de la larga lista de desafíos a enfrentar para mejorar y normalizar la educación de estos niños y niñas afectados por el conflicto (UNHCR, 2015).

La reconstrucción del sistema educativo, sea formal o no formal, ofrece a los menores y a sus familias una cierta esperanza y más seguridad (Selby y Kagawa, 2012; Sinclair, 2002; Uddin, 2015). La simple rutina de ir a la escuela y el trabajo con adultos de confianza en un entorno de apoyo y tolerancia, ayuda a los menores afectados por conflictos armados a recuperarse del trauma. Los beneficios de la educación son duraderos porque los menores transferirán a la siguiente generación todo lo que hayan adquirido (Linden y Alzira, 2011; Sinclair, 2002; World Humanitarian Summit Secretariat, 2015).

$\mathrm{Al}$ mismo tiempo, las alteraciones que conlleva una crisis o conflicto armado pueden ser percibidas como una oportunidad para el cambio. En otras palabras, una oportunidad para instalar nuevos valores y mejores sistemas educativos, con la intención de ayudar a los niños y niñas a mejorar su autoestima y esperanza con el fin de prepararlos para que sean parte activa en la sociedad del futuro (Bird, 2009; Nicolai y Triplehorn, 2003).

En esta investigación se parte de que las Tecnologías de la Información y de la Comunicación (TIC) ofrecen distintas posibilidades para solucionar las dificultades mencionadas. Las TIC no solo son útiles para mejorar el acceso a la educación en las aulas, sino también fuera de ellas. Además, pueden ser la base para coordinar y mejorar notablemente el rendimiento de actores claves en la reconstrucción de los sistemas educativos afectados por una crisis.

El presente artículo forma parte de una investigación sobre el papel de las TIC en la reconstrucción de la educación en países afectados por conflictos armados. Se centra en presentar los resultados que la investigación ha obtenido en cuanto al uso de las redes sociales y la mensajería instantánea en los tres casos que forman parte de la investigación que se está desarrollando. 


\section{Revisión de la literatura}

\subsection{Impacto de los conflictos armados en la educación en Siria}

Según varios estudios, los conflictos armados pueden perturbar o destruir totalmente las condiciones necesarias para asegurar un entorno sano, estable y seguro para la educación. Los conflictos armados afectan de forma directa el acceso a la educación y causan una serie de consecuencias negativas de las cuales citamos entre otras: la muerte o el desplazamiento del profesorado y el alumnado, la destrucción total o parcial de las escuelas y las infraestructuras educativas, la amenaza a la seguridad de los niños y niñas, la pérdida de datos, la interrupción completa o parcial de la comunicación entre los actores principales de la educación (Barry y Newby, 2012; CICR, 2011; Manuchehr, 2011; Mendenhall, 2014; Montjourides, 2013; Poirier, 2012; Shemyakina, 2011).

Teniendo en cuenta lo anterior, es preciso hacer un resumen de la situación previa de la educación en Siria, que permita contextualizar los efectos del conflicto. Antes del inicio del conflicto en marzo del 2011, Siria tenía un récord destacable en relación con los datos de la educación básica. Un $97 \%$ de los niños y niñas en edad de ir a la escuela primaria estaban asistiendo a la escuela, y un $67 \%$ de los niños y niñas en edad de la escuela secundaria. El índice de alfabetización era elevado, más del 90\% de la población, hombres y mujeres incluidos. Este índice superaba el de países vecinos como el Líbano, Jordania e Iraq. (McDonald et al., 2017; Sirin y Rogers-Sirin, 2015; UNICEF, 2013; Watkins, 2013).

Sin embargo, a medida que se extendía el conflicto, la tasa neta de inscripción a la educación primaria, que todavía estaba en el 98,9\% en 2009, disminuyó a menos del $71 \%$ en 2013 (figura 1). En el 2016, Siria había perdido más de la cuarta parte de sus escuelas, más de 6.000 escuelas resultaron dañadas por la violencia, forzadas a cerrar o utilizadas para combatir o refugiar a cientos de familias desplazadas (UNICEF, 2016a). Desde el inicio del conflicto, el ambiente educativo se deterioró rápidamente; afectando de forma grave la vida y el futuro de muchos niños y niñas (UNESCO, 2016).

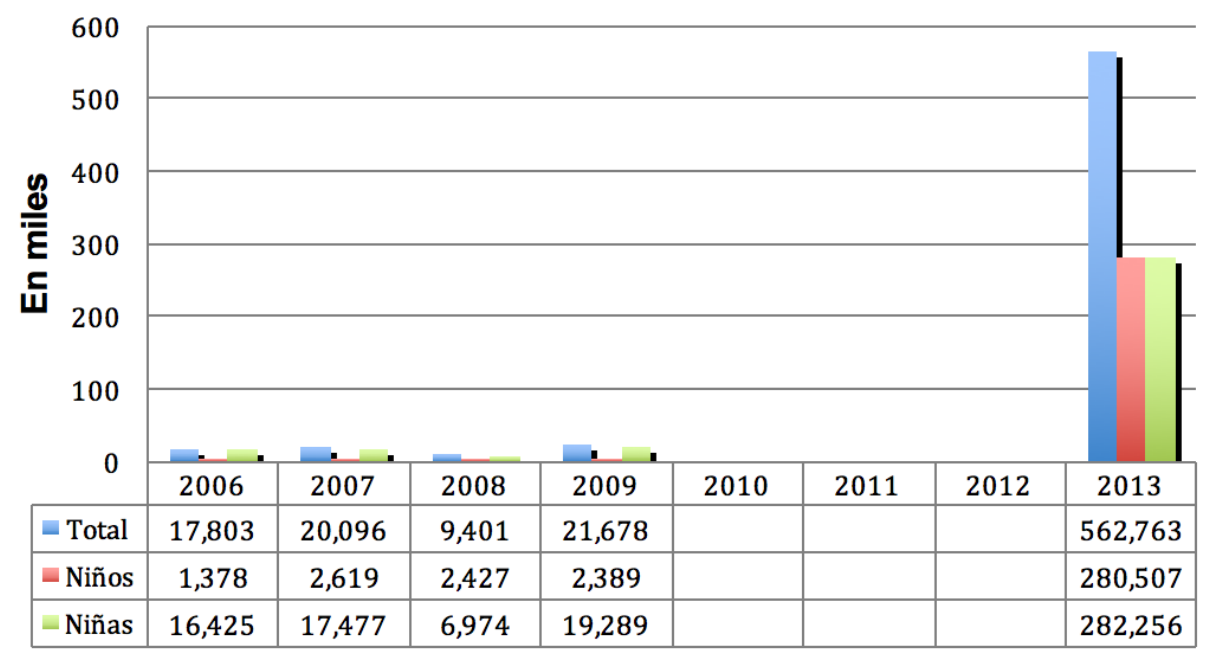

Figura 1. Número de niños y niñas sirios fuera de la escuela en miles Fuente: Elaboración propia a partir de ACNUR (2013).

El conflicto armado en Siria ha causado una de las peores crisis humanitarias y de refugiados en la historia (Berti, 2015; UNHCR, 2014), con más de cinco millones de 
refugiados (5,605,366 refugiados registrados) y más de seis millones de desplazados internos (casi la mitad son menores, según los datos actualizados de ACNUR el 18 de agosto de 2018 en su página web oficial ${ }^{1}$ )

\subsection{Las tecnologías de la información y de la comunicación y la educación en contextos de conflicto armado y de emergencia}

En las últimas décadas, las TIC han venido desempeñando un papel importante en la educación en situaciones de emergencia y de conflictos armados. El uso de las TIC tiene el potencial de mejorar el rendimiento y la respuesta de los gobiernos y organizaciones responsables de asegurar soluciones rápidas y sostenibles para los problemas de la educación causados por estas situaciones excepcionales de emergencia. Algunos ejemplos de este uso pueden observarse en proyectos como eLearning Sudan (eLS) en Sudán, Enza en Kenya (Dahya, 2016), EDC'S Shaqoodoon program en Somalia, o los cursos de formación a distancia ofrecidos por IASC-UNITAR (Barry y Newby, 2012) y también la plataforma Riff diseñada para la recogida de datos en tiempo real durante desastres del tipo que sean (Coyle y Meier, 2009).

Las TIC no sólo pueden posibilitar la educación, en términos del acceso a los procesos de enseñanza y aprendizaje, sino que implican también la coordinación y la comunicación entre las diferentes partes implicadas, como los trabajadores humanitarios y representantes del gobierno, y entre profesorado y padres y madres, por ejemplo (Dahya, 2016; Semaan, 2011).

\subsection{Facebook y WhatsApp en la educación}

Se entiende que las redes sociales y las aplicaciones de mensajería instantánea ofrecen un abanico de ventajas al sector educativo, tanto en la educación a distancia como presencial. Los expertos confirman que aumentan la participación del alumnado y mejoran tanto el trabajo colaborativo como autónomo. Estas herramientas ayudan a promover un ambiente de aprendizaje y colaboración más interactivo y atractivo. También ayudan a desarrollar nuevas competencias, mejoran las relaciones sociales entre los miembros de los grupos, y aumentan la motivación en el aprendizaje. Otra ventaja sería la mejora de la autoestima y la libertad de expresión (Alonso et al., 2014; Cabero-Almenara y Marín-Díaz, 2014; Vaquerizo, 2012).

De la revisión literaria (Barhoumi, 2015; Bouhnik, Deshen y Gan, 2014; Iglesias, González Díaz y González Guerrero, 2016; Monguillot, González Arévalo y Guitert, 2017; Nassar, 2015; Wasserman y Zwebner, 2017) se ha podido constatar que existen dos herramientas dominantes, la mensajería instantánea de WhatsApp y la red social Facebook, incluyendo Facebook Messenger. El uso de ambas herramientas representa una situación de win-win (todos ganan), es decir, tanto la institución académica, como el profesorado, los estudiantes y los padres y madres.

Estas herramientas representan una innovadora estrategia de cooperación en el proceso de aprendizaje (Cabero-Almenara y Marín-Díaz, 2014), que permite la construcción de conocimiento en grupo y la mejora de la comunicación (Padrón, 2013) además de mejorar la relación profesorado-estudiante (Alonso et al., 2014; Nedungadi, Mulki y Raman, 2018).

\footnotetext{
${ }^{1}$ http://www.unhcr.org/syria-emergency.html
} 


\section{Método}

La investigación se desarrolló como un estudio de caso múltiple de carácter cualitativo que permitió conceptualizar y hacer seguimiento de tres casos (Flick, 2014; Marshall y Rossman, 2016). La investigación tiene también un enfoque etnográfico virtual (Marshall y Rossman, 2016; Savin-Baden y Tombs, 2017), ya que hacemos un seguimiento de las interacciones personales y profesionales de identidades en la red haciendo una observación del espacio virtual donde se producen estas interacciones.

\section{Población, muestra y muestreo}

La población de muestra de la investigación son los tres casos de estudio y sus integrantes (niños y niñas sirios, cuadro académico, padres y madres, directores de proyectos, expertos y expertas en educación en conflicto y en acción humanitaria durante un conflicto armado, voluntarios y voluntarias y trabajadores sociales, entre otros). El primer criterio para la selección de los casos fue que hubiera sido pionero en pensar y ofrecer soluciones al problema de la educación interrumpida de los niños y niñas sirios. Aunque posteriormente surgieran otras iniciativas y proyectos similares.

En segundo criterio a tener en cuenta en la selección fue que cada caso representara un modelo diferente. Así, el primero es una plataforma en línea de recursos educativos abiertos (REA); el segundo está diseñado específicamente para refugiados sirios y ofrece educación de forma presencial y en línea en el Líbano; y el tercero opera dentro y fuera de Siria, colaborando con el gobierno sirio y con los gobiernos de países de acogida vecinos, este último ofrece educación y formación de forma presencial y en línea, además ofrece consultoría y tutoría al Ministerio de Educación Sirio de forma directa. A continuación, se ofrece una descripción de cada caso:

1) Nafham (Comprendemos en Árabe, www.nafham.com): Se trata de la primera plataforma en línea en el mundo árabe que ofrece recursos educativos abiertos (REA) tanto útiles para la educación formal como no formal. El contenido educativo consta de vídeos educativos en línea (Youtube) de una duración de entre cinco y quince minutos que cubren el plan de estudios formal de Siria, Egipto, Arabia Saudita, Kuwait y Argelia. Los vídeos están disponibles de forma gratuita en línea a través de la página web, y a través de aplicaciones móviles (Android, iOS y Windows). Del total de los vídeos disponibles, aproximadamente 1000 cubren el plan de estudios sirio.

2) Jusoor (Puentes en árabe, www.jusoorsyria.com): Jusoor es un programa de educación para refugiados diseñado después del inicio del conflicto y como respuesta a la crisis de la educación, dirigido a los niños y niñas refugiados en el Líbano. Jusoor cuenta con más de 3000 niños y niñas en sus escuelas. Tiene como objetivo garantizar a los refugiados sirios en el Líbano acceso a la educación primaria formal, además de ofrecer un fuerte apoyo psicosocial en el marco de la participación comunitaria y la rehabilitación.

3) UNICEF (Fondo de las Naciones Unidas para la infancia, https://uni.cf/1oXD12g): Su labor se focaliza en hacer frente a todas las necesidades de niños y niñas, incluida la educación. También da apoyo a la formación del profesorado y al equipamiento de las aulas. En el caso de Siria, UNICEF colabora con varios socios a escala local, nacional e internacional, incluso con el gobierno sirio y con los gobiernos vecinos 
de acogida para garantizar a los niños y niñas sirios sus derechos básicos, entre los cuales se encuentra el acceso a la educación.

\section{Técnicas e instrumentos de recogida de datos}

Para realizar la investigación, se han utilizado diferentes técnicas de recogida de datos para garantizar la calidad y la validez de las conclusiones de este estudio (Flick, 2014; Hamilton y Corbett-Whittier, 2013). Con el fin de preparar el marco conceptual y establecer los bases iniciales de la investigación, se ha realizado una revisión literaria extensa sobre temas como la educación en situaciones de emergencia y conflicto armado, educación para la paz y prevención de conflicto y TIC en la educación, entre otros.

La investigación se ha basado en dos técnicas de investigación cualitativa. La primera ha sido la entrevista semi-estructurada en profundidad con informantes claves (cuadro académico, padres y madres sirios, niños y niña sirios, directores de proyectos, expertos y expertas en educación en conflicto y en acción humanitaria durante un conflicto armado, voluntarios y voluntarias y trabajadores sociales). Las entrevistas se basaron en la conversación y el diálogo entre la investigadora principal y el entrevistado o entrevistada.

Para planificar y llevar a cabo las entrevistas, se han tenido en cuenta varios elementos y factores, sea en cuanto a la metodología del entrevistador o la sensibilidad del tema en cuestión, la situación personal de los entrevistados, sobre todo a la hora de hablar con menores de edad. Se han realizado un total de 26 entrevistas con una duración de entre 40 a 60 minutos con informantes claves de los casos de estudio. Previamente, se han realizado otras 7 entrevistas en profundidad, de tipo exploratorias con, i) expertos en ayuda humanitaria y educación en países en conflicto, que han servido para ofrecer pautas generales sobre el impacto de los conflictos armados en general y sobre el caso de Siria en particular; y ii) expertos y trabajadores del sector educativo en situaciones de emergencia.

Las entrevistas se han realizado directamente con el/la entrevistado/a sin la intervención de intérprete, y se han realizado en árabe, francés e inglés. Todas las entrevistas han sido grabadas, transcritas y traducidas al español por parte de la investigadora principal. Para localizar los informantes claves y definir su perfil, se han utilizado las redes sociales como Facebook y LinkedIn, además de contactos personales de la investigadora principal, y se han enviado correos electrónicos a los directivos y responsables de los proyectos casos de estudio para conseguir el permiso de entrevistar otros informantes claves dentro de cada caso. La mayoría de los informantes entrevistados han sido sugeridos por parte de los directores de los proyectos casos de estudio. y los demás han sido localizados por la investigadora principal durante la fase de trabajo de campo y gracias a la observación realizada en la red social Facebook. Del total de las entrevistas, 7 se han realizado de forma presencial cara a cara, y el resto se ha realizado en línea con la ayuda de las herramientas TIC como Skype, Google Hangout, WhatsApp Call, Facebook Call, Viber Call, entre otras.

La segunda técnica de recogida de datos utilizada en esta investigación ha sido la observación, sea en línea o de forma presencial durante las visitas de la investigadora principal a los campos de refugiados en el Líbano. En la era digital, la observación en línea se considera como un método de investigación cualitativo. La observación que se ha llevado a cabo se describe como pasiva, donde el investigador participa de forma discreta e interviene en las discusiones pocas veces, como definen Savin-Baden y Tombs (2017). La observación ha durado todo el período de la investigación entre los años 2013 y 2018, y ha servido para la localización y la selección de los casos al inicio, para entender la 
situación en cuestión, para identificar a posibles agentes claves, para contrastar los datos recogidos en la literatura con la realidad y para la recogida de datos multimedia como texto, capturas de pantalla, vídeos y fotos de las complejas interacciones en la situación en cuestión. Se ha pedido a algunos de los agentes claves, el consentimiento para compartir capturas de pantalla de su uso de WhatsApp.

El trabajo de campo presencial que se desarrolló en el Líbano durante el curso escolar 2012-2013 y 2017-2018, se organiza en dos partes, la primera con las visitas a los diferentes campos de refugiados en el Líbano, y la segunda con la asistencia a las clases impartidas dentro del caso de estudio Jusoor. En cuanto al trabajo de campo en línea, ha consistido en la observación de 7 cuentas (grupos y páginas Facebook) de los casos de estudio que están detallados en el cuadro 1.

Cuadro 1. Listado de los grupos y páginas observados en Facebook

\begin{tabular}{cll}
\hline \multicolumn{1}{c}{ CASO } & \multicolumn{1}{c}{ NoMBRE } & \multicolumn{1}{c}{ ENLACE } \\
\hline UNICEF* & $\begin{array}{l}\text { UNICEF Middle East and } \\
\text { North Africa }\end{array}$ & https://www.facebook.com/UNICEFmena/ \\
\hline UNICEF* $^{*}$ & UNICEF Lebanon & https://www.facebook.com/UNICEFLebanon/ \\
\hline UNICEF $^{*}$ & UNICEF in Arabic & https://www.facebook.com/unicef.in.arabic/ \\
\hline Jusoor* & $\begin{array}{l}\text { Jusoor - Syrians Forward } \\
\text { Together }\end{array}$ & https://www.facebook.com/jusoor.syria/ \\
\hline Jusoor** & $\begin{array}{l}\text { Jusoor Refugee Education } \\
\text { Program }\end{array}$ & https://www.facebook.com/groups/jusoor.rep/ \\
\hline Nafham* & Nafham Education & https://www.facebook.com/NafhamEducation/ \\
\hline Nafham** & Crowd Teaching & https://www.facebook.com/groups/nafham.live/ \\
\hline
\end{tabular}

Nota: * Página web. ** Grupo cerrado.

Fuente: Elaboración propia.

\section{Análisis de datos}

Tal como lo precisa Merriam (1988), el análisis de los datos comienza con el primer documento leído, con la primera entrevista y con la primera observación. Todo detalle e información servirá para complementar el cuadro final de la investigación. Toda la información y los datos obtenidos a lo largo de la investigación han sido organizados en carpetas en la nube en Dropbox. Para efectuar el análisis de los resultados se ha utilizado un método mixto, en papel, de manera manual y con la confección de tablas para localizar la información relevante en relación a las diferentes categorías, y en la parte final de la investigación se ha utilizado el software de análisis cualitativo AtlasTi. Inicialmente y gracias a la revisión literaria y la primera toma de contacto con el campo de trabajo, se identificaron categorías iniciales sobre la educación en Siria, los desafíos y obstáculos para la educación en caso sirio, prácticas y usos TIC presentes (figura 2).

No obstante, y especialmente después de haber comenzado con las entrevistas y la observación, han emergido otras categorías que no estaban previamente establecidas y han llevado a una diferente lectura de los datos. Por ejemplo, la categoría de comunicación digital no estaba establecida y se ha podido confirmar que es una práctica común en los tres casos. Otros temas relacionados al entorno social y cultural tampoco estaban en el foco de interés y aparecieron posteriormente. 


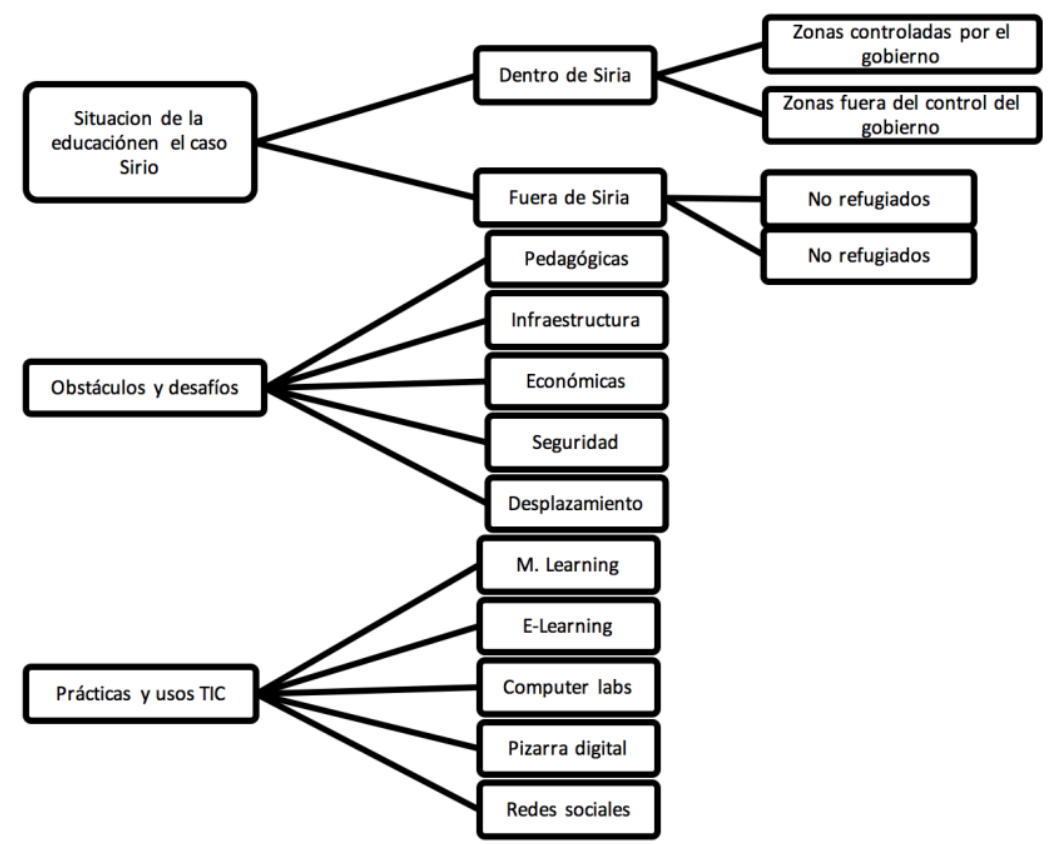

Figura 2. Mapa conceptual de las primeras categorías establecidas Fuente: Elaboración propia.

Las categorías han surgido del análisis de las transcripciones de las entrevistas, del material multimedia recolectado, de los textos digitales, de las notas tomadas a lo largo de la investigación y por supuesto de la revisión literaria. Así que finalmente, se ha podido organizar la información en tres categorías marco y otras subcategorías en cada categoría marco. Estas categorías son: las TIC en el proceso de enseñanza-aprendizaje; las TIC para la comunicación y coordinación y difusión de la información y, por último, las TIC para la recopilación y gestión de datos.

\section{Resultados}

En este trabajo, se presenta una parte del análisis de los datos obtenidos, que son en cuanto al uso de Facebook y WhatsApp, organizados según las tres categorías marco: proceso de enseñanza y aprendizaje, comunicación, coordinación e información, y recopilación y gestión de datos.

\subsection{Categoría 1: Proceso de enseñanza y aprendizaje}

Se han identificado varios usos académicos de las dos herramientas WhatsApp y Facebook, que se pueden agrupar en las siguientes subcategorías:

\subsubsection{Formación a distancia}

En los casos Nafham y Jusoor, se utiliza la función de transmisión en vivo de Facebook, la cual permite asistir a una clase o formación desde cualquier lugar y deja un espacio de interactividad para los participantes. Los alumnos y alumnas pueden también formular preguntas en formato de comentarios o en el chat. Los profesores y los estudiantes han podido tener una interacción cara a cara sin que importe su ubicación geográfica. 
También, ha servido para la formación de voluntarios locales en sesiones sobre el trabajo social con expertos en psicología y gestión de crisis. A parte de reducir las distancias, esta función permite reducir los costes ya que en la mayoría de los casos no se puede asumir el pago del desplazamiento y alojamiento del experto o de los participantes.

En el caso Jusoor, por ejemplo, se hacen a menudo sesiones en vivo con expertos en diferentes campos, en Facebook se anuncia la sesión por la página de Jusoor y luego se realiza la difusión en vivo. Las preguntas de los participantes se hacen en los comentarios y se responden también en la sección de los comentarios. Las sesiones suelen ser en vivo en Facebook y Youtube a la vez, como se aprecia en la figura 3.

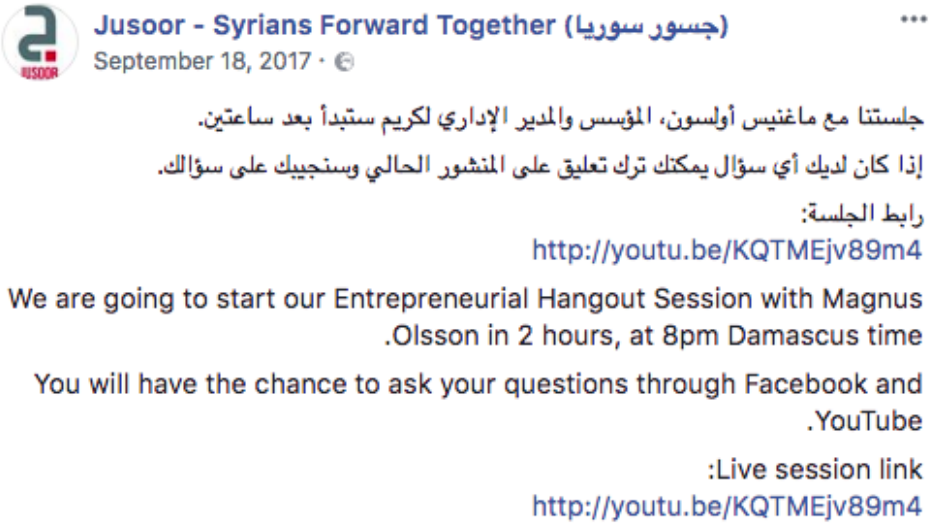

Figura 3. Recordatorio de la sesión formativa con un experto en gestión Nota: Se anuncia que los participantes pueden formular sus preguntas a través de Facebook y Youtube.

Fuente: Recuperado de la página de Jusoor.

\subsubsection{Trabajo colaborativo}

En los tres casos de estudio se utiliza la función de grupos en WhatsApp, los cuales se utilizan sobre todo entre estudiantes para ponerse de acuerdo en los trabajos en grupo solicitados en clase. De esta manera, WhatsApp permite estructurar mejor el trabajo en grupo y enriquecerlo, dado que todos pueden comentar, aunque no estén en el aula o en un mismo espacio físico concreto. Es la herramienta más utilizada para consultar dudas de clase a los compañeros. WhatsApp permite interactuar a cualquier hora y desde cualquier sitio, por lo que ese dinamismo es aprovechado por los alumnos y alumnas para preguntarse dudas.

Por ejemplo, TK, alumna en $3^{\circ}$ de primaria que usa el móvil de su madre para comunicarse con sus compañeras de clase en la escuela de Beirut gestionada por Jusoor: "Hablamos de muchas cosas por el grupo de WhatsApp, pero a mí me escriben sobre todo mis compañeras para que les explique cosas que hemos aprendido en clase, sobre todo de inglés porque soy muy buena". De esta manera, WhatsApp fomenta el aprendizaje colaborativo y ayuda en la construcción del conocimiento entre todos tal como indican los estudios de Gewerc-Barujel, Montero-Mesa y Lama-Penín (2014) o Marín, Negre y Pérez (2014).

\subsubsection{Aprendizaje de idiomas}

En el caso Jusoor, la función de mensajes de voz en WhatsApp es utilizada para grabar tareas relacionadas con el aprendizaje de idiomas, lo que permite al profesorado o pares 
evaluar la expresión y la pronunciación oral del alumno o la alumna, tal como lo confirma ST, la directora del programa de educación para los refugiados en el del proyecto Jusoor. El proyecto es en colaboración con dos instituciones para la enseñanza de inglés a los refugiados, en línea, utilizando la aplicación WhatsApp. Las dos instituciones son Education Development Trust (www.educationdevelopmenttrust.com), dentro del programa Enseñanza de la lengua inglesa y Voxy (www.voxy.com), una plataforma para aprender inglés en línea.

WhatsApp se utiliza para enviar y recibir las actividades y tareas, se hace un seguimiento personalizado y por grupos, además de hacerse la evaluación de los aprendizajes. Se utilizan los mensajes de voz para la evaluación del nivel. La figura 4 muestra las tareas creadas y compartidas por la profesora de inglés en el grupo de profesores en la escuela de Jusoor en Beirut.

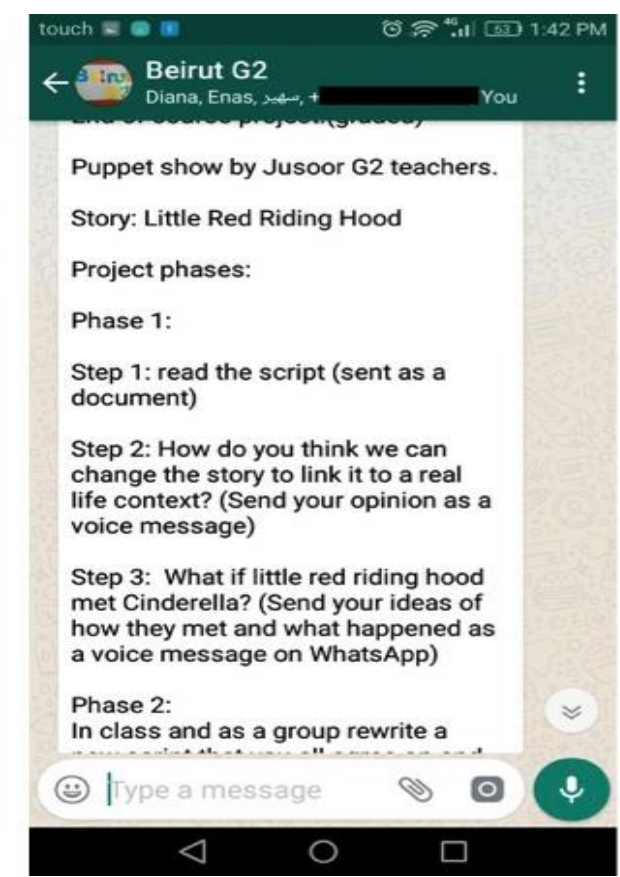

Figura 4. Ejemplo de las tareas compartidas por WhatsApp Fuente: Elaboración propia.

La tarea se divide en dos fases. En la primera fase hay tres pasos. El primer paso trata sobre la lectura de un fragmento del cuento de Caperucita Roja que ha sido compartido previamente en el grupo de WhatsApp. El segundo paso requiere que den su opinión sobre como cambiarían el texto, tarea que hacen en inglés vía mensaje de voz. En el último paso se les pide que digan qué pasaría si Caperucita roja se encontrara con Cenicienta, opinión que envían también en inglés y por mensaje de voz. En la segunda fase del ejercicio se forman grupos y tienen que escribir juntos una nueva versión del cuento para presentar al resto de la clase. De esta manera, el grupo de WhatsApp se convierte en un aula de aprendizaje y todos los miembros pueden escuchar las respuestas de todos.

\subsection{Categoría 2: Comunicación, coordinación e información}

En esta categoría se agrupan los usos no directamente relacionados con el proceso de enseñanza y aprendizaje, no son temas directamente pedagógicos, pero son necesarios para que el proceso de enseñanza en sí mismo pueda llevarse a cabo. 


\subsubsection{Difusión de la información}

En los tres casos se ha detectado que Facebook está siendo usado como plataforma de difusión y comunicación de la información. Los objetivos detrás de las campañas organizadas en Facebook son sobre todo para sensibilizar a la comunidad internacional e invitar a los internautas a promover acciones de apoyo a los refugiados y afectados. La acción puede ser un comentario, un compartir, un "me gusta" o hasta llegar a donar y apoyar económicamente la causa. La figura 5, por ejemplo, representa una captura de pantalla del vídeo compartido por UNICEF sobre el niño refugiado sirio Fares. Fares tiene seis años y trabaja más de ocho horas al día, nunca ha estado en la escuela y no sabe leer ni escribir. UNICEF pretende llamar la atención sobre el tema del trabajo infantil y el analfabetismo que son, de hecho, algunas de las consecuencias directas del conflicto armado.

Otro ejemplo en el caso Nafham, dónde MG, director de contenido del proyecto, utiliza Facebook para "pasar mensajes sobre temas de género, aprovechar la presencia de los jóvenes en las redes sociales para sensibilizarles sobre las igualdades y el derecho de las niñas a la educación”.

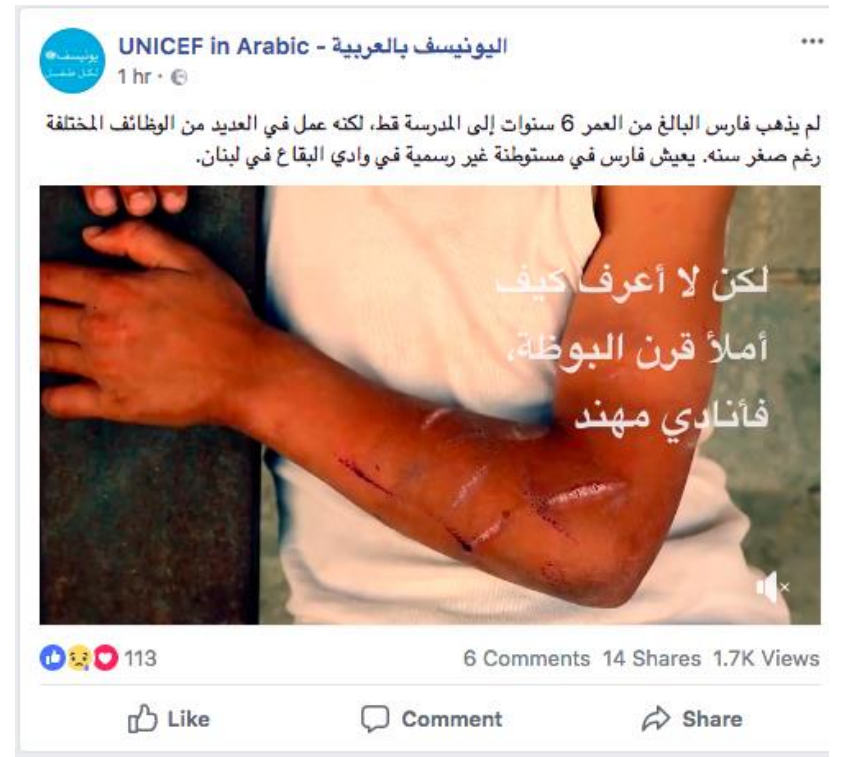

Figura 5. Página de Facebook de UNICEF del niño refugiado sirio en el Líbano Fares Fuente: Captura del vídeo recuperado de www.youtube.com/watch?v=jBz83K8-Yuk.

\subsubsection{Organización y coordinación}

Un uso común en los tres casos estudiados, es el uso de WhatsApp o Facebook, para concretar y confirmar reuniones de trabajo entre los miembros de la misma organización con miembros de otras organizaciones o con individuos concretos. Los grupos de WhatsApp permiten organizar a los trabajadores y voluntarios en un sitio y coordinar las acciones que hay que emprender en tiempo real.

En la figura 6, por ejemplo, que es una captura de pantalla del grupo de WhatsApp de los profesores y la dirección dentro del proyecto Jusoor; el director comparte los resultados trimestrales con el profesorado y les felicita por la evolución de los alumnos y alumnas. 
También avisa de la ausencia de dos profesoras y pide una sustitución para el turno de noche.

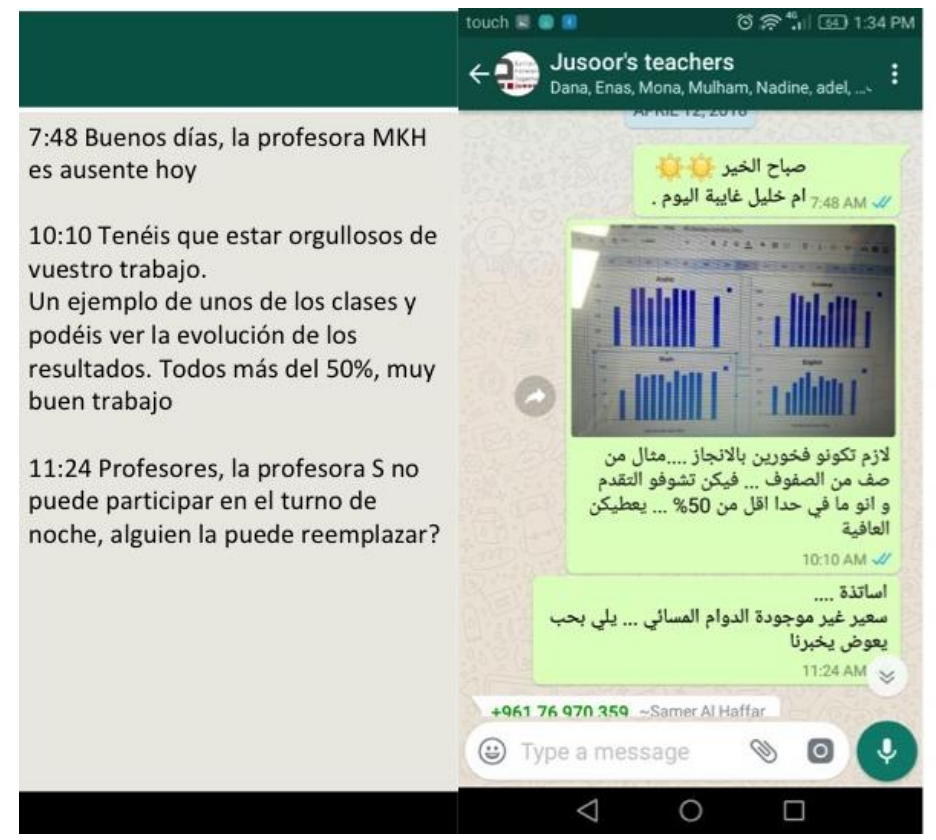

Figura 6. Grupo de WhatsApp de los profesores y la dirección en el proyecto Jusoor

Fuente: Elaboración propia a partir de la captura de pantalla de la conversación.

\title{
3.2.3. Comunicación y avisos
}

El tema de la seguridad es uno de los temas más importante en el conflicto sirio: la seguridad de los niños y niñas, sus padres, sus docentes y sus escuelas. Facebook se ha convertido en la fuente más rápida de noticias y de información sobre los ataques. La administración de la escuela o de la institución comparte avisos sobre la seguridad en tiempo real como, por ejemplo, suspender las clases, dar información sobre ataques por un mortero contra la institución, dar los nombres de las víctimas sobre todo si hay menores involucrados, entre otros. La maestra de árabe HM de una escuela gestionada por UNICEF en Damasco es un ejemplo cuando dice:

\begin{abstract}
Hemos creado un grupo cerrado para nuestra escuela para avisar a los alumnos y a los padres de cualquier incidencia, sobre todo en los años 2013 y 2014 que es cuando estábamos viviendo los peores momentos de esta guerra. Esto forma parte del plan de emergencia que hemos implementado en el colegio. Si hay alguna noticia de peligro, se hace tipo cadena, utilizamos sms, WhatsApp y Facebook.
\end{abstract}

En el mismo contexto, muchos de los padres y madres en los campos de refugiados son analfabetos y no saben leer ni escribir. La mensajería de voz de WhatsApp ofrece una solución perfecta para la administración, si quieren avisar a los padres y madres sobre cualquier asunto relacionado con sus hijos e hijas. Por ejemplo, información sobre las notas, una reunión, alguna emergencia, o también si hace falta la firma de los padres para aprobar algo como, por ejemplo, una visita al museo o salir fuera del campo de refugiados. Cada responsable lee el contenido de la carta y lo graba como mensaje de voz de WhatsApp para enviarlo, y el padre o la madre, lo pueden aprobar o no, contestando en forma de mensaje de voz también, como si fuera una firma digital oral. 


\subsection{Categoría 3: Recopilación y gestión de datos}

Como se ha visto en otras publicaciones (Montjourides, 2013), uno de los hándicaps que dificultan la labor de las organizaciones y gobiernos trabajando en el campo de la ayuda humanitaria en general, y en la educación en situaciones de emergencia en particular, sería la falta de datos y estadísticas, además de la dificultad, y a veces, la imposibilidad de acceder a los datos durante un conflicto armado. No obstante, es crucial poder conseguir los datos, poder analizarlos y tratarlos para organizar y planificar las acciones futuras. El caso sirio no es ninguna excepción, pero el desarrollo y el avance de las TIC pueden mejorar la recogida y la gestión de estos datos. En este apartado, se presentan las prácticas detectadas en relación con el uso de WhatsApp y Facebook para mejorar este aspecto.

\subsubsection{Recopilación de datos}

Los tres casos estudiados confirman la utilización de la herramienta de encuestas en línea de Facebook. Esta función permite preguntar a los seguidores de las páginas o miembros de grupos en Facebook para obtener retroalimentación o su opinión sobre algún tema. Esto permite tanto recoger datos sobre las opiniones y preferencias del público, como reforzar el diálogo y la interacción entre los usuarios. Por ejemplo, en la figura 7 vemos a MG, director de contenido en Nafham publicando una encuesta en el grupo cerrado Crowd Teaching que reúne a los voluntarios y creadores de contenido en Nafham. Pregunta a los miembros su opinión sobre qué temas hay que priorizar para ofrecer un mejor servicio en Nafham.

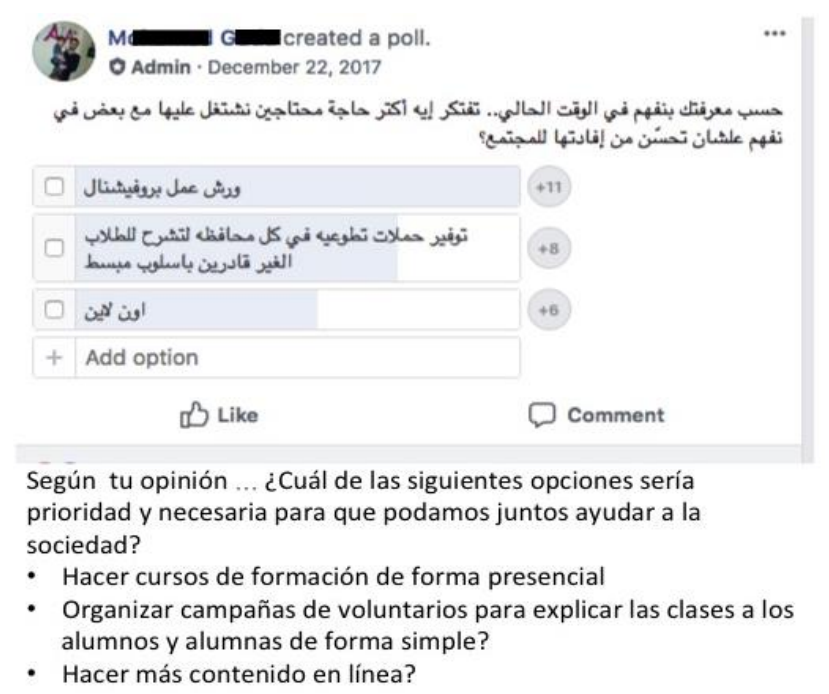

Figura 7. Encuesta en el grupo cerrado Crowd Teaching en Facebook Fuente: Elaboración propia a partir de la encuesta publicada por Nafham.

En el mismo contexto, y como consecuencia de las sanciones aplicadas contra Siria por parte de la Unión Europea, Estados Unidos y otros países, el acceso o el uso de sistemas de gestión de datos es casi imposible ya que es imprescindible tener un espacio en uno de estos países para almacenar los datos. Esto hace que el trabajo de recogida y gestión de datos dentro de Siria sea difícil. Los trabajadores locales y los voluntarios, intercambian datos e información sobre la situación de la educación en varias zonas de Siria a través Facebook y WhatsApp. Por ejemplo, información sobre los daños sufridos en una escuela, el número de niños y niñas fuera de la escuela, información sobre el material que falta en 
alguna escuela o centro educativo. Esta información se descarga y se comparte posteriormente con las personas responsables de analizar las necesidades y de planificar las acciones a realizar. Como se ve en el testimonio de HS, responsable de informática en la oficina de UNICEF en Damasco cuando dice:

\begin{abstract}
Intento documentar todo el trabajo que hacemos en UNICEF, a qué zonas hemos llegado, cuántas personas, cuántos niños y niñas, cuántos hombres y cuántas mujeres, cuántas madres y cuántos padres, todo tipo de información que pueda tener lo registro en este programa para poder ayudarnos a planificar nuestras futuras acciones. En base a esto, hago estudios y análisis. Esto nos ayuda a ver las carencias que tenemos, las zonas que necesitan más ayuda, qué tipo de ayuda se necesita, qué tipo de acciones hay que hacer depende del grado de la situación. Con los voluntarios y trabajadores de las organizaciones socios, me comunico sobre todo por WhatsApp y me mandan todos los datos por WhatsApp como, por ejemplo, fotos de las encuestas, fotos del material distribuido, vídeos de los niños y niñas además de informes y evaluaciones en formato de grabación de voz. To me encargo de recoger estos datos, introducirlos en formato hoja de Excel y posteriormente en el programa que he creado para poder elaborar los informes necesarios para nuestro trabajo.
\end{abstract}

\title{
3.3.2. Gestión de datos
}

Todos los casos usan plataformas de análisis de datos, los cuales recogen en línea con el fin de medir y analizar el impacto de las publicaciones y la interacción de la comunidad con ellas. La plataforma más usada es Google Analytics. Esto les permite evaluar las estrategias y medidas actuales para planificar y organizar las futuras. Estas mismas plataformas permiten extraer informes y datos en formato Excel que suelen ser compartidos con otros departamentos de la misma organización con el fin de elaborar informes internos y otros públicos. RAD, responsable de las redes sociales en UNICEF Líbano, confirma esta apreciación cuando afirma que los informes generados son altamente importantes para los responsables del departamento de comunicación y les ayudan a mejorar su trabajo. Dice,

Nos dimos cuenta de que los usuarios no miran los videos que son más de un minuto y 20 segundos en Facebook, antes había un malentendido de concepto y solíamos poner videos de 5 minutos de duración. Pues nadie los miraba. Ahora la situación es diferente, hemos aprendido y ahora llegamos a transmitir el mensaje de forma eficaz. $\Upsilon$ todo gracias a los informes generados sobre el impacto de las acciones hechas por nuestra parte en redes sociales.

\section{Discusión y conclusiones}

De lo anterior, se puede avanzar una parte de los resultados de la investigación en relación con el uso de Facebook y WhatsApp con el fin de asegurar la educación a los niños y niñas afectados por el conflicto sirio como sigue:

Tanto Facebook como WhatsApp resultan relevantes para diferentes fines entre los cuales citamos: acceder a la educación de forma móvil tanto a la educación formal como no formal; mejorar la comunicación; mejorar el trabajo colaborativo y en grupo; intercambiar documentos y material multimedia de forma segura y eficaz; permitir un espacio de interacción entre los padres y el equipo docente o administrativo para resolver asuntos administrativos o de seguridad en situaciones de emergencia; reforzar las relaciones personales entre los alumnos y alumnas; permitir un espacio de escucha para aquellos y aquellas que sufren acoso o que están en situación de trauma; ahorrar gastos y acercar distancias, entre otros. 
Los resultados concuerdan con lo que otros estudios sostienen sobre el inmenso potencial que las redes sociales tienen en el proceso de enseñanza-aprendizaje fuera de la escuela en situaciones de crisis y de conflicto armado, y en particular, Facebook y WhatsApp que ya son las herramientas más utilizadas entre los refugiados en el caso de Siria (Dahya, 2016; Mason y Buchmann, 2016). No obstante, hemos constatado que no todos están sacando provecho a la totalidad de las posibilidades que ofrecen estas herramientas, sobre todo en cuanto al proceso de enseñanza y aprendizaje, tal como lo plantea el estudio de Miao y otros (2018).

De lo presentado, se puede concluir y afirmar que Facebook y WhatsApp están siendo usados por parte de los agentes claves en los casos de estudio, sean padres o madres, docentes, directores y responsables de proyectos, niños y niñas, además de voluntarios y trabajadores sociales, no solamente con fines pedagógicos o en el proceso enseñanzaaprendizaje, sino también con fines comunicativos y de coordinación. Asimismo, se están utilizando para la difusión de información relevante sobre la educación y la crisis de la educación siria, y para la recopilación y gestión de datos.

También se utiliza como herramienta de recogida de datos ya que se pueden tomar fotos, hacer vídeos, grabaciones de voz y mandarlos de forma inmediata a las sedes siempre que haya conexión a internet. Es una forma de documentar los hechos, y sirve en la recogida de datos necesarios para la planificación de la acción humanitaria en relación con la educación y también para la evaluación del funcionamiento de las acciones ofrecidas en un determinado momento.

Asimismo, confirmamos que Facebook se usa para promover los valores, servicios, eventos y otras actividades que ofrecen cada uno de los casos estudiados en esta investigación. También se usa para recaudar fondos, aunque no siempre de forma directa. Por todo lo presentado, podemos decir que la innovación en el caso de nuestra investigación no tiene que ver con la creación o la elaboración de herramientas tecnológicas avanzadas y/o específicas para el caso sirio, sino más bien en hacer un uso innovador de las herramientas existentes con la población objetivo.

Como conclusión final podemos decir que hace falta más investigación aún para descubrir todas las posibilidades que ofrecen Facebook y WhatsApp y también otras aplicaciones, para apoyar la educación en contextos de crisis y conflicto armado.

\section{Referencias}

Alonso, C., Bosco, A., Corti, F. y Rivera, P. (2014). Prácticas de enseñanza mediadas por entornos 1x1: Un estudio de casos en la educación obligatoria de Cataluña. Profesorado. Revista de Currículum y Formación del Profesorado, 18(3), 99-119.

Barhoumi, C. (2015). The effectiveness of WhatsApp mobile learning activities guided by activity theory on students' knowledge management. Contemporary Educational Technology, 6(3), 221-238.

Barry, B. y Newby, L. (2012). Use of technology in emergency and post-crisis situations. París: IIEPUNESCO.

Berti, B. (2015). The Syrian refugee crisis: Regional and human security implications. Strategic Assessment, 17(4), 89-125. 
Bird, L. (2009). Promoting resilience: Developing capacity within education systems affected by conflict. París: UNESCO.

Bouhnik, D., Deshen, M. y Gan, R. (2014). WhatsApp goes to school: Mobile instant messaging between teachers and students. Journal of Information Technology Education, 13, 217-231. https://doi.org/10.1016/j.iheduc.2016.06.001

Cabero-Almenara, J. y Marín-Díaz, V. (2014). Educational possibilities of social networks and group work. University students' perceptions. Comunicar, 21(42), 165-172. https://doi.org/10.3916/C42-2014-16

CICR. (2011). Los niños afectados por los conflictos armados y otras situaciones de violencia. Ginebra: CICR.

Coyle, D. y Meier, P. (2009). New technologies in emergencies and conflicts: The role of information and social networks. Londres: UN Foundation-Vodafone Foundation Partnership.

Dahya, N. (2016). Education in conflict and crisis: How can technology make a difference? A landscape review. Bonn: GIZ.

Flick, U. (2014). An introduction to qualitative research. Londres: SAGE.

Gewerc-Barujel, A., Montero-Mesa, L. y Lama-Penín, M. (2014). Colaboración y redes sociales en la enseñanza universitaria. Comunicar, 5(1), 53-63. http://doi.org/10.3916/C42-2014-05

Hamilton, L. y Corbett-Whittier, C. (2013). Using case study in education research. Londres: SAGE.

Iglesias, M., González Díaz, C. y González Guerrero, L. (2016). Los grupos de Facebook como herramienta de enseñanza-aprendizaje en la universidad. En R. Roig-Vila (Ed.), Tecnología, innovación e investigación en los procesos de enseñanza-aprendizaje (pp. 676-683). Madrid: Octaedro.

Linden, J. y Alzira, M. (2011). Beyond literacy: Non-formal education programmes for adults in Mozambique. Compare: A Journal of Comparative and International Education, 41(4), 467-480. https://doi.org/10.1080/03057925.2011.581514

Manuchehr, T. (2011). Education right of children during war and armed conflicts. Procedia. Social and Behavioral Sciences, 15, 302-305. https://doi.org/10.1016/j.sbspro.2011.03.090

Marín, V., Negre, F. y Pérez, A. (2014). Entornos y redes personales de aprendizaje (PLE-PLN) para el aprendizaje colaborativo. Comunicar, 21, 35-43. https://doi.org/10.3916/C42-2014o3

Marshall, C. y Rossman, G. B. (2016). Designing qualitative research. Los Angeles, CA: SAGE.

Mason, B. y Buchmann, D. (2016). ICT4Refugees. A report on the emerging landscape of digital responses to the refugee crisis. Recuperado de https://goo.gl/ZPiymy

McDonald, A., Buswell, M., Khush, S. y Brophy, D. M. (2017). Invisible wounds. The impact of six years of war on the mental health of Syria's children. Londres: Save the Children.

Mendenhall, M. A. (2014). Education sustainability in the relief-development transition: Challenges for international organizations working in countries affected by conflict. International Journal of Educational Development, 35, 67-77. https://doi.org/10.1016/j.ijedudev.2012.08.006

Merriam, S. B. (1988). Case study research in education: A qualitative approach. Londres: Jossey-Bass.

Miao, F., Pagano, M., Atchoarena, D., Pimmer, C., Gröhbiel, U., Zelezny-Green, R., ... Strecker, J. (2018). A lifeline to learning. Leveraging technology to support education for refugees. Recuperado de https://goo.gl/AKQPz2 
Monguillot, M., González Arévalo, C. y Guitert, M. (2017). El WhatsApp como herramienta para la colaboración docente. Revista Digital de Educación Física, 44, 56-62.

Montjourides, P. (2013). Education data in conflict-affected countries: The fifth failure? Londres: Springer.

Nassar, D. A. (2015). Using Social network as an education enhancement tools a case study of using WhatsApp in Princess Nourah University. Journal of Educational \& Instructional Studies in the World, 6, 18-21.

Nedungadi, P., Mulki, K. y Raman, R. (2018). Improving educational outcomes \& reducing absenteeism at remote villages with mobile technology and WhatsApp: Findings from rural India. Education and Information Technologies, 23(1), 113-127. https://doi.org/10.1007/s10639-017-9588-Z

Nicolai, S. y Triplehorn, C. (2003). The role of education in protecting children in conflict. Network Paper: Humanitarian Practice Network, 42, 1-36.

No Lost Generation. (2016). Syria crisis education strategic. Londres: SAGE.

Padrón, C. J. (2013). Estrategias didácticas basadas en aplicaciones de mensajería instantánea WhatsApp exclusivamente para móviles. Eduweb, 7(2), 123-134.

Poirier, T. (2012). The effects of armed conflict on schooling in Sub-Saharan Africa. International Journal of Educational Development, 32(2), 341-351. https://doi.org/10.1016/j.ijedudev.2011.06.001

Savin-Baden, M. y Tombs, G. (2017). Research methods for education in the digital age. Londres: Bloomsbury Publishing Plc.

Selby, D. y Kagawa, F. (2012). Disaster risk reduction in school curricula: Case studies from thirty countries. París: UNICEF.

Semaan, B. C. (2011). Recovery, resilience and beyond: ICT use during ongoing disruption (Tesis doctoral). Universidad de California Los Ángeles, Estados Unidos.

Shemyakina, O. (2011). The effect of armed conflict on accumulation of schooling: Results from Tajikistan. Journal of Development Economics, 95(2), 186-200. https://doi.org/10.1016/j.jdeveco.2010.05.002

Sinclair, M. (2002). Planning education in and after emergencies. Fundamentals of education and training. París: UNESCO.

Sirin, S. R. y Rogers-Sirin, L. (2015). The educational and mental health needs of Syrian children. Washington, DC: MPI.

Uddin, A. (2015). Education in peace-building: The case of post-conflict chittagong hill tracts in Bangladesh. The Oriental Anthropologist, 15(1), 59-76.

UNESCO. (2016). Global education monitoring report 2016. Education for people and planet: Creating sustainable futures for all. París: UNESCO.

UNHCR. (2014). Syria regional response plan (RRP6). París: The UN Refugee Agency.

UNHCR. (2015). The challenge of education. París: The UN Refugee Agency.

UNICEF. (2013). Syria crisis: Education interrupted. Amman: UNICEF.

UNICEF. (2016a). No place for children. The impact of five years of war on Syria's children and their childhoods. Amman: UNICEF.

UNICEF. (2016b). Syria crisis: Education fact sheet. Five years of crisis and conflict. Amman: UNICEF. 
UNICEF. (2017). Syria crisis. 2017 Humanitarian results. Amman: UNICEF.

Vaquerizo, M. B. (2012). Enseñanza-aprendizaje con web 2.0 y 3.0. Vivat Academia, 117, 116-121. https://doi.org/10.15178/va.2011.117E.116-121

Wasserman, E. y Zwebner, Y. (2017). Communication between teachers and parents using the WhatsApp application. International Journal of Learning, Teaching and Educational Research, 16(12), 1-12. https://doi.org/10.26803/ijlter.16.12.1

Watkins, K. (2013). Education without borders. Londres: EwB.

World Humanitarian Summit Secretariat. (2015). Restoring humanity, global voices calling. Nueva York, NY: United Nations.

\section{Breve CV de las autoras}

\section{Mirey Alfarah}

Experta en educación en países afectados por conflicto armado y en educación y tecnología. Titular de dos Masters en educación y comunicación por la Universidad Autónoma de Barcelona. Actualmente es candidata al doctorado de educación en la Universidad Autónoma de Barcelona, e investiga el tema de la educación en países en zonas afectadas por conflicto armado y el papel de las Tecnologías de información y de comunicación (TIC). En particular el caso Siria. Nacida en Damasco, Siria 1980. ORCID ID: https://orcid.org/o000-0003-3515-4045. Email: mirey.alfarah@e-campus.uab.cat

\section{Alejandra Bosco}

Profesora Titular de Tecnología Educativa en el área de Didáctica y Organización Escolar, del Departamento de Pedagogía Aplicada de la Universitat Autònoma de Barcelona. Miembro del grupo de investigación consolidado Equipo de desarrollo Organizacional - EDO - del Dpto. de Pedagogía Aplicada. Coordinadora el grupo \&quot;DIM- EDAINET: Entornos de Aprendizaje, Innovación Educativa y Tecnologías\&quot;, grupo de investigación reconocido por la Universidad Autónoma de Barcelona desde 2001. Las principales investigaciones que ha desarrollado giran en torno al potencial educativo de las tecnologías digitales en el desarrollo del currículum en todos los niveles educativos de la educación formal, pero también en la educación no formal, y en la vida cotidiana. A este ámbito es al que corresponden la mayor parte de sus publicaciones. ORCID ID: http://orcid.org/0000-0002-1825-0097. Email: alejandra.bosco@uab.cat 